\title{
Uncertainties in partial duration series modelling of extremes related to the choice of the threshold value
}

\section{Santiago Beguería}

UCEL - Utrecht Centre for Environment and Landscape Dynamics - Utrecht University. PO Box 80115, 3508 TC Utrecht, The Netherlands

s.begueria@geog.uu.nl

\begin{abstract}
Partial duration series modelling is a robust tool with which to model hydrologic extremes, but because of several technical problems it remains underused. The most important obstacle is the choice of the threshold value, a matter that is currently under investigation by several authors. The truncation value affects the basic assumptions of the model, including arrival times and exceedance magnitudes. This paper considers changes in parameter and quantile estimates as a function of the threshold value (sampling variability). Simulated and real data were used to test the consistency of the model, and a new modelling procedure based on increasing threshold censoring is proposed to overcome these problems. Poisson arrivals and Generalized Pareto exceedances were assumed, and the suitability of this model at different threshold values has been also addressed.
\end{abstract}

Keywords: frequency analysis; extreme rainfall events; peaks over threshold; partial duration series; threshold value; Generalized Pareto distribution; threshold stability 


\section{Introduction}

The magnitude - frequency analysis of hydrologic extremes (e.g. floods and extreme rainfall events) relates event magnitudes with their frequency by using curve-fitting techniques and instrumental data on the studied variable. This allows estimation of the frequency of events greater than those observed during the period of record. The frequency associated with an event of given magnitude $x$ is expressed conventionally in Hydrology by the concept of return period. The return period of an event $\left(T_{x}\right)$ can be defined as the average number of observations to be made to obtain one observation equalling or exceeding its magnitude $(X \geq x)$. This includes the following underlying assumptions:

1) The extremes are a random variable, and thus can be described by a probability distribution.

2) This distribution does not change from sample to sample (homogeneity).

3) The data are independent.

Two methods are normally used to sample the original data for extreme events: annual maximum (AM) and partial duration (PD) series. AM series are composed of the greatest events of each year from a particular period, so the series length equals the number of years of recording. As the events have been sampled at fixed intervals, the return period of an event of magnitude greater or equal to $x$ equals the inverse of its probability of exceedance: $T_{x}=1 /$ $\mathrm{p}(X \geq x)$; the probability that an event of magnitude $x$ will occur at least once in a period of $m$ years is: $\mathrm{p}(n \geq 1 \mid m, x)=1-[1-\mathrm{p}(X \geq x)]^{m}$. According to a now well stablished theorem originally due to Fisher and Tippett (1928), a series of sample maxima like AM series are described by a Generalised Extreme Value distribution, what includes the well known Gumbel, Fréchet and Weibull distributions. The AM approach has been very popular, as demonstrated by the large number of works in which it is used (e.g. Chow, 1964; Alexander et al., 1969; Kirkby, 1969; Yevjevich, 1972; Potter, 1987; Bobeé and Ashkar, 1991; García-Ruiz et al., 2000).

On the other hand, in PD (also known as peaks or exceedances over threshold) analysis the samples are not collected at fixed intervals. PD series are type I censored series; that is, they 
include all the values of the variable that exceed an a priori determined threshold, $x_{0}$, what defines the transformed variable $Y$ :

$Y=X-X_{0}$

for every case where $X>x_{0}$. The difference between the two approaches is illustrated in Figure 1. As demonstrated by Pickands (1975), if $X$ is an iid variable a threshold value $x_{0}$ can be found that makes the process converge to a Generalised Pareto distriution (GP). This includes as a special case the Exponential distribution, that has long been very popular in PD modelling (see Cunnane, 1973).

In contrast to AM series, where the sampling interval for an observation is known, in the PD approach the frequency of the events in a given time period (involving the inter-arrival times and the number of occurrences) is random. The simplest model, and thus the most widely used, is to assume a Poisson process for event arrivals (Cunnane, 1979). Under the Poisson assumption, the return period of an exceedance of magnitude $y$, in years, is easily calculated as: $T=1 /[\lambda \cdot \mathrm{p}(Y \geq y)]$, where $\lambda$ is the average number of occurrences per year. Examples of its use can be seen in Todorovic and Zelenhasic (1970), Ashkar and Rousselle (1987), or Rosbjerg et al. (1992).

When dealing with the right tails of frequency distributions, accurate estimation of extreme events is severely limited by the lack of sufficient information (García-Ruiz et al., 2000). The PD approach has several important advantages over the AM approach, as it adapts better to heavy-tailed distributions (Madsen et al., 1997b) and makes a more efficient use of information since it permits to include more cases (Kite, 1977; Chow et al., 1988; Madsen et al., 1997b). Indeed, a common objection to the AM approach is that it systematically ignores the 2nd, 3rd, etc. annual registrations that can exceed the maximum events of other years and be considered extreme as well. The PD approach avoids these problems by considering all the events above a certain level. This level is fixed by the modeller and provides a more physicallybased definition of what must be considered an extreme event. Besides, it controls the size of 
the resulting sample, allowing one to include more cases. Cunnane (1973) has demonstrated that PD series are more efficient at parameter estimation than AM series when the average number of annual occurrences is above 1.6, and exceedances are modelled by an exponential distribution. Madsen et al. (1997a; 1997b) arrived at similar conclusions for both at-site and regional modelling.

Despite these advantages, the PD approach is still not widely used. This is probably due to some unsolved technical problems and the lack of a generally accepted methodology for selecting the truncation value $x_{0}$. This is a very relevant problem, as several important features of frequency modelling are highly sensitive to the value selected. The value of $x_{0}$ is directly related to the assumption of independence of arrival times and exceedances. In general, a high truncation value ensures that the events are independent, but can lead to an important loss of information and increasing uncertainty. On the other hand, a low truncation value can make the events too close in time, thus introducing serial dependence of both occurrence times and magnitudes, thereby violating the assumption of independence.

The truncation value can also affect the assumption of homogeneity, since different hydrological or meteorological mechanisms can prevail at different frequencies. Adamowski (2000) suggested that the truncation value can even affect the unimodal or multimodal character of the distribution, hiding the presence of different generating processes at different frequencies. A basic condition in PD modelling is that the model will remain stable even if the threshold varies, but this key feature is not well fixed.

Finally, even if the process agrees with all the assumptions, the truncation value directly controls the size of the sample. The methods for extreme variables curve-fitting are very sensitive to the data used and small changes of the truncation value can significantly modify the lower part of the distribution. Minor changes on the left tail of the empirical distribution of the extremes can lead to great differences in the parameter estimates, especially with threeparameter distributions. Önoz and Bayazit (2001), for example, found different frequency 
estimations depending on the selected threshold, demonstrating the need for more detailed studies.

The choice of the truncation value is frequently based on expert judgement, a procedure that involves a great level of subjectivity. Lang et al. (1999) reviewed different more systematic methods for the choice of the threshold value. The simplest one is to choose the truncation value according to a fixed frequency. Different values have been suggested, but the most frequently cited correspond to an event that repeats 1.2 to 5 times a year. Ashkar and Rousselle (1987) have defined the optimum value as the minimum $x_{0}$ that makes the exceedance series fit a Poisson process. Rosbjerg and Madsen (1992) proposed a standardized procedure based on a predefined factor $k$ and the mean and standard deviation of the original series: $x_{0}=\bar{x}+k \cdot s$; the authors recommend a value of $k$ around three.

Notwithstanding these different proposals, the uncertainty in PD analysis related to the selection of the threshold value has never been thoroughly addressed. In this paper the sensitivity of different aspects of PD modelling to the value of the truncation level $\left(x_{0}\right)$ is analysed. Two sets of 100 simulated series have been generated for hypothesis testing, as well as real data from twelve meteorological stations in the period 1941-1991. The modelling framework and the tests used are described in Section 2. They include the modelling of exceedance frequency by the Poisson distribution, and the use of the GP distribution for modelling exceedance magnitudes. In Section 3 are presented the results of the tests on both arrival times and exceedance magnitudes. In Section 4, the results are discussed and an alternative modelling procedure is proposed based on increasing threshold censoring instead of a unique or discrete truncation value. This new approach is tested upon the simulated and observed data sets.

\section{Partial duration series modelling}

\subsection{The Poisson - Generalised Pareto model}


One of the features of partial duration series analysis is the modelling of exceedance frequencies; that is, the number of occurrences within a given time period. The most simple and generally used assumption is that the occurrence of events exceeding the given threshold $x_{0}$ follows a Poisson process (Cunnane, 1979). Given a discrete variable $N$, expressing the annual number of occurrences of a given event, the number $n$ of events occurring in any year constitutes a Poisson variate, with probability:

$$
P(N=n \mid \lambda)=\mathrm{e}^{-\lambda} \cdot \frac{\lambda^{n}}{n !}, \quad \mathrm{n}=1,2, \ldots
$$

The frequency parameter $\lambda$, equalling the average number of exceedances per year, can be estimated from the sample mean: $\hat{\lambda}=\bar{n}$. The Poisson assumption implies that the occurrences are independent, i.e. the probability of observing an extreme event in time $t+j$ does not depend on the occurrence of an extreme event in time $t$.

This property is not so obvious when dealing with natural processes. Frequently, hydrological phenomena such as rainfall or floods show a tendency to appear grouped in bunches, introducing a variable degree of serial dependence in the data series. In this case a declustering process is normally applied to the data.

As it has been said above, GP is the limit distribution for a type I censored variate, and has been selected by numerous authors for PD modelling (Van Montfort and Witter, 1986; Hosking and Wallis, 1987; Wang, 1991; Madsen and Rosbjerg, 1997; Martins and Stedinger, 2001). The GP distribution is described by a shape parameter $\kappa$, a scale parameter $\alpha$, and a location parameter $\xi$, and has the following cumulative distribution function:

$$
\begin{array}{ll}
P(X \leq x \mid \xi, \alpha, \kappa)=1-\left(1-\kappa \cdot \frac{(x-\xi)}{\alpha}\right)^{\frac{1}{\kappa}}, & \kappa \neq 0 \\
P(X \leq x \mid \xi, \alpha, \kappa)=1-\exp \left(-\frac{x-\xi}{\alpha}\right), & \kappa=0
\end{array}
$$


The GP distribution contains the exponential distribution as a special case, when $\kappa=0$ (second expression). For $\mathrm{k}<0$ the distribution is long-tailed, and for $\kappa>0$ it becomes upper-bounded with endpoint at $-\alpha / \kappa$, and should be used with caution unless there is physical evidence of upper bounding.

Since in PD modelling the lower bound (truncation value) is known, there is no need to estimate the location parameter $\xi$. The former expression can be adapted to the transformed variate $Y$ :

$$
\begin{array}{ll}
P(Y \leq y \mid \alpha, \kappa)=1-\left(1-\kappa \cdot \frac{y}{\alpha}\right)^{\frac{1}{\kappa}}, & \kappa \neq 0 \\
P(Y \leq y \mid \alpha, \kappa)=1-\exp \left(-\frac{y}{\alpha}\right), & \kappa=0
\end{array}
$$

The estimates of GP parameters, $\hat{\alpha}$ and $\hat{\kappa}$, can be obtained by different procedures. Here I describe the method of probability-weighted moments (Hosking and Wallis, 1987):

$$
\begin{aligned}
& \hat{\alpha}=\hat{\lambda}_{1} \cdot\left(\frac{1}{\hat{\tau}_{2}}-1\right) \\
& \hat{\kappa}=\frac{1}{\hat{\tau}_{2}}-2
\end{aligned}
$$

where the ratio $\hat{\tau}_{2}=\hat{\lambda}_{2} / \hat{\lambda}_{1}$ is the L-coefficient of variation. $\lambda_{1}$ and $\lambda_{2}$ (L-mean and Lstandard deviation) are the two first L-moments, which are linear functions of the probability weighted moments (PWMs) of the sample, and can be generated by the function:

$$
\lambda_{r}=\sum_{k=0}^{r-1}(-1)^{r-k} \cdot\left(\begin{array}{l}
r \\
k
\end{array}\right) \cdot\left(\begin{array}{c}
r+k \\
k
\end{array}\right) \cdot \beta_{k}=\sum_{k=0}^{r-1}(-1)^{r-k} \cdot \frac{r !}{k ! \cdot(r-k) !} \cdot \frac{(r+k) !}{k ! \cdot r !} \cdot \beta_{k}
$$

The order $r$ PWM, $\beta_{r}$, can be obtained from the sample using the unbiased estimator of Landwehr et al. (1979):

$$
\hat{\beta}_{r}=n^{-1} \cdot \sum_{i=1}^{n}\left(\begin{array}{c}
i-1 \\
r
\end{array}\right) \cdot\left(\begin{array}{c}
n-1 \\
r
\end{array}\right)^{-1} \cdot y_{(i)}=n^{-1} \cdot \sum_{i=1}^{n} \frac{(i-1) \cdot(i-2) \ldots(i-r)}{(n-1) \cdot(n-2) \ldots(n-r)} \cdot y_{(i)}
$$


$i$ being the rank of the observation after sorting the data by ascending order: $y_{1} \leq y_{2} \leq \ldots \leq y_{n}$.

Under the Poisson assumption, the T-year exceedance $Y_{T}$ is defined as the $(1-1 / \lambda T)$ quantile in the distribution of the exceedances. Inverting equation (4) and substituting for $X$, we obtain the following expression for the T-year event $X_{T}$, expressed in the original scale:

$$
\begin{array}{ll}
X_{T}=x_{0}+\frac{\alpha}{\kappa} \cdot\left[1-\left(\frac{1}{\lambda \cdot T}\right)^{\kappa}\right], & \kappa \neq 0 \\
X_{T}=x_{0}+\alpha \cdot \log \left(\frac{1}{\lambda \cdot T}\right), & \kappa=0
\end{array}
$$

An important property of the GP model is the threshold stability: If $Y=X-x_{0}$ is a GPdistributed variable with given shape parameter $\kappa$, it continues to be GP-distributed with identical shape parameter for any higher truncation value $x_{0}+q$. The new frequency parameter $\lambda_{x_{0}+q}$ is obtained as:

$$
\lambda_{x_{0}+q}=\lambda \cdot p(X \geq q)
$$

The new scale parameter $\alpha_{x_{0}+q}$ is also related to the new truncation value (Madsen et al., 1997b):

$$
\alpha_{x_{0}+q}=\alpha-\kappa \cdot q
$$

The asymptotic stability of the shape parameter and the scaling of the scale parameter with the variation of $x_{0}$ ensure the stability of quantile estimates, which is a basic premise of exceedance frequency analysis.

Another related property of the GP distribution refers to the mean excess: If $Y=X-x_{0}$ is a GPdistributed variable, then the mean excess over threshold $x_{0}+q$ is:

$$
E\left(Y \mid x_{0}+q\right)=\frac{\alpha_{x_{0}+q}-\kappa \cdot q}{1+\kappa}
$$


for $\kappa>-1$. This implies that $E\left(Y \mid x_{0}+q\right)$ is linear in $x_{0}$ with gradient $m$ :

$$
m=\frac{-\kappa}{1+\kappa}
$$

\subsection{Assessing the sensitivity to the truncation value}

As explained before, the choice of a truncation value to construct the series of exceedances is the first step in the PD approach. The choice of the threshold value affects several crucial aspects of PD modelling, including the assumptions of homogeneity and independence. These consequences have been addressed in both arrival times and exceedance magnitudes, applying different tests.

First of all, the truncation value affects the independence of arrival times. Hydrological series are not normally a real random process, but it is generally considered that a threshold can be found that is high enough to ensure the independence of the occurrences. The suitability of the Poisson assumption has been tested by means of the Dispersion Index (DI) statistic (Cunnane, 1979):

$$
D I=\frac{s^{2}}{\hat{\lambda}}
$$

where $s^{2}$ is the estimated variance of $N$. If the data adjust to a Poisson process then the mean and the variance of $N$ should be approximately equal; hence, $D I$ is expected to be 1 . Confidence levels for $D I$ can be calculated by testing against a chi-square distribution with $M-1$ degrees of freedom, $M$ being the total number of years in the sample. The Poisson hypothesis is not rejected if the estimated $D I$ is within the range of $\left(\chi_{\alpha / 2, M-1}^{2} /(M-1), \chi_{1-\alpha / 2, M-1}^{2} /(M-1)\right)$, where $\alpha$ is the significance level. The $D I$ ratio has been calculated for all the data series using increasing truncation values. 
The suitability of the GP model for the real data series at diferent threshold values can been tested by the mean excess plot. It plots the average excess over a thresholds against the value of the threshold, for a given set of threshold values. As it has been explained above, if the variable follows a GP distribution over a threshold value $x_{0}$, then the mean excess plot should appear approximately linear at that point. This property can also help in selecting the most convenient threshold value, that should be the lowest one for which the GP model holds.

The adequacy of the GP distribution has been also tested by means of the L moment plot. This is a plot of the sample $\left(\hat{\imath}_{3}, \hat{\tau}_{4}\right)$ L-moments estimates $\left(\tau_{3}=\lambda_{3} / \lambda_{2} ; \tau_{4}=\lambda_{4} / \lambda_{2}\right)$, for a given set of increasing threshold values. A curve representing the L-moment ratios of the GP distribution is also plotted, using the approximation:

$$
\tau_{4}=\frac{\tau_{3} \cdot\left(1+5 \cdot \tau_{3}\right)}{5+\tau_{3}}
$$

(Hosking, 1990). The deviations from this theoretical curve can help in deciding the range of applicability of the model.

Another important aspect concerning the modelling of exceedance magnitudes is the sampling variation of parameter and quantile estimates with the variation of the truncation value. Despite the asymptotic threshold stability property of PDS/GP, that guarantees the stability of the model at different thresholds, in practice a certain level of uncertainty is to be expected when working with finite (and, very often, short) data series. This is uncertainty is expressed as a sampling random error in parameter and quantile estimates, and it is expected to increase as does the truncation value. This fact has been addressed by estimating parameters and quantiles for the series generated by the same set of threshold values cited above. The value corresponding to a 25 year recurrence interval has been arbitrarily selected to assess the stability of the final predictions, but the same results have been found for higher centiles. 


\subsection{Data used}

Real data of daily rainfall registers from twelve climatic stations during the period 1941-1991 was used. The rain gauge stations are distributed along a mountainous area in the Central Spanish Pyrenees between the Gállego (east) and the Esca (west) rivers, and from the main divide, coinciding with the French border, to the southern limit of the Spanish Pyrenees (Fig. 3). The climate is dominated by Atlantic influences from the northwest and Mediterranean fluxes from the south and southeast. Annual precipitation decreases from north to south and from west to east. It averages 2,500 $\mathrm{mm}$ at high altitudes (Rijckborst, 1967; García-Ruiz et al., 1985), decreasing to $600-800 \mathrm{~mm}$ in the Inner Depression.

A common problem when dealing with natural metheorological and hydrological data is that they normally present high serial correlation, with threhold exceedances occurring grouped in clusters. For this reason a declustering scheme has been applied to the data series. Each series of alternative days with values exceeding the initial threshold $\mathrm{x} 0$ was considered a cluster. From each cluster only the daily peak (maximum) was conserved.

Additionally, two sets of simulated data series were generated for hypothesis testing. Each simulation consisted in one hundred series of 2000 elements of GP-distributed exceedances over $\mathrm{X}=0$. Two data sets with $\kappa=-0.1$ and -0.2 where generated, with common scale parameter $\alpha=15$. The corresponding 25-year recurrence events are 146.58 and $218.20 \mathrm{~mm}$ (eq. 8).3.

\section{Results}

\subsection{Arrival times}

Figure 4 shows the evolution of the DI ratio related to the threshold value in the real data set. Figure 4A represents the original daily rainfall series, and 4B shows the results of the cluster maxima series. The shaded area is the confidence interval for $D I=1$ at a 0.95 confidence level. 
It is clear that the original daily series did not adjust to a Poisson process. Even that they showed a tendence towards the confidence region, they only reached it at the highest treshold values. This confirms the high importance of the serial dependence present in the data set. After the declustering process, however, the series fitted perfectly the Poisson assumption of DI $=1$, even at low values of the threshold level, so this was the data set used for the reminder of the analysis.

\subsection{Exceedance magnitudes}

Figure 4 shows the mean excess plots of the 12 rainfall series. In general, a clear linear pattern is visible in all the series from the lowest values of the threshold, until the highest values where the plot becomes unstable. As explained before, this supports the hypothesis of the GP model for the data. A more detailed mean excess plot is showed in figure 5 for the station of Pineta. In the superior axis is shown the mean frequency of exceedances over the threshold. A regression line has been added to the mean excess plot, until the value of $x_{0}=40 \mathrm{~mm}$, showing a $\mathrm{r}^{2}$ of 0.956. Using eq. 11 , the slope of the line $(m=0.1037)$ yields an estimation of $\kappa=-0.0940$. This represents a prior estimation of the shape parameter of the GP process, that coincides quite well with the posterior PWM estimation $(\kappa=-0.1358)$.

The results from the $\mathrm{L}$ moment plots, shown in figure 6 , confirm the convenience of the GP model. A similar pattern was found in all the data series, the points describing elliptical trajectories around the GP curve, that appeared clearly as an atractor of the process until the highest values of the threshold. Only the station of Seira showed a distribution of points far from the GP curve, what questions the suitability of the model for that station.

The preceding results confirm that the series of cluster maxima follow quite well the Poisson-GP model. However, the variability due to the sampling error can lead to uncertainty in parameters and quantiles. The results concerning the estimation of parameters and quantiles at different thresholds are shown in figures 7 to 9 . The results of the GP simulation, which 
represent the theoretical model, are shown in figure 7. As can be seen, the average value of $\kappa$ remained constant and very close to the correct values of -0.1 (A) and -0.2 (B) along the whole range of threshold values. As expected, the variance of the estimates was significatly higher in the $\kappa=-0.2$ simulation, as this implies a higher tail and hence higher sample variability. The average value of $\hat{\alpha}$ increased regularly in both cases, in relation to the rise in $x_{0}$, as stated by Eq. 10. The evolution of these two parameters guaranteed the stability of quantile estimations, yielding a constant average value of $X_{25}$ around 146.6 (9A) and 218.2.

If the average values tended to be invariant with respect to the threshold value, the variance of the estimates varied greatly in the GP simulation (Fig. 7). Both $\hat{\alpha}$ and $\hat{\kappa}$ showed increasing variance, implying an increasing uncertainty in parameter estimation as the threshold grew, especially from a threshold of $40 \mathrm{~mm}$ on. Despite this, the variance of the $X_{25}$ estimates was more or less constant along the range of threshold values (Fig. 9A, 9B), 95\% of the predictions lying within the range $123.92-177.35 \mathrm{~mm}$ for $\kappa=-0.1$ and $242.40-266.05$ for $\kappa=-$ 0.1 .

Figures 8 and 9B also show the fluctuation of parameters and quantiles related to the changes in the threshold value of a real data series, corresponding to the station of Pineta. As can be seen, the shape parameter estimates $(\hat{\kappa})$ experienced large and random fluctuation between the extremes of -0.36302 and 0.333 . In general, sudden changes in $\hat{\kappa}$ were compensated by associated changes in $\hat{\alpha}$, which kept the $X_{25}$ estimates relatively stable (Fig. 9B). Nevertheless, variation in the quantile estimates was also noticeable, between extremes of 104.0 and $68.7 \mathrm{~mm}$.

Pineta station has been shown here as an example, but very similar results were found in the rest of the real series. Some statistics on the performance in the estimation of $X_{25}$ are shown in Table 1, covering both the simulated and the real data sets. As well as the average values of $X_{25}$, the standardized mean bias error (SMBE) and the root mean square error (RMSE) are given for the two simulations, that can be compared with the theoretically expected value. The same 
does not hold for the real data, so the extremes of $X_{25}$ and the coefficient of variation are given as expressing the degree of uncertainty in the estimation of quantiles. The simulations showed a RMSE of 16.2 for $\kappa=-0.1$ and 29.7 for $\kappa=-0.12$. The coefficient of variation ranged between 0.04 and 0.10 for the real data series, comparable to the values of 0.057 and 0.060 obtained in the two simulations.

\section{Discussion and proposal of a new method}

With respect to the arrival times, the real data series behaved as a random Poisson process even at lower values of the threshold. This contrasts with the original series before declustering, that did not reache the confidence limit for a Poisson process until the highest centiles, or did not reach it at all.

The GP model was found to be valid for the real series of exceedances, as demonstrated by the mean excess and L moment ratio plots. Despite this, a great level of uncertainty has been found in the process of parameter and quantile estimation. This uncertainty seems to be inherent to the process, and must be attributed to small changes in the lowest part of the sample introduced by the changes in the threshold value. This fact was observed in the whole range of threshold values for the two simulations, and only showed a relatively small increase at the highest centiles. Thus, a single threshold value cannot be recommended for accurate modelling.

In contrast to the common approach to PD modelling, based on a unique threshold value, an increasing threshold censoring procedure is proposed. Taking advantage of the properties of the GP distribution, the final $\hat{\alpha}_{x_{0}}^{x_{n}}$ and $\hat{\kappa}_{x_{0}}^{x_{n}}$ parameters are averaged over the range of threshold values $\left(x_{0}, x_{n}\right)$. The $\alpha$ parameter is reparametrized according with eq. 10 to be consistent with its initial value:

$$
\hat{\alpha}_{x_{0}}^{x_{n}}=\sum_{i=0}^{n} \hat{\alpha}_{x_{i}}+\hat{k}_{x_{0}}^{x_{n}} \cdot\left(x_{i}-x_{0}\right)
$$


being $\hat{\kappa}_{x_{0}}^{x_{n}}$ the average of the $\kappa$ parameter for the range of thresholds $\left(x_{0}, x_{n}\right)$. The resulting expression for quantile $X_{T_{x_{0}}}^{x_{n}}$ is:

$$
X_{T x_{0}}^{x_{n}}=x_{0}+\frac{\hat{\alpha}_{x_{0}}^{x_{n}}}{\hat{\kappa}_{x_{0}}^{x_{n}}} \cdot\left[1-\left(\frac{1}{\lambda_{0} \cdot T}\right)^{x_{x_{0}}^{x_{n}}}\right]
$$

where $x_{0}$ is the initial threshold value and $\lambda_{0}$ the initial frequency.

The lower limit of the range of thresholds, $x_{0}$, is the lowest value for wich the Poisson-GP model holds, according to the diagnostic tools provided here (DI, mean excess and L moment ratios plots). The upper limit of the threshold range can also be derived from theese plots, taking the point where the process starts to be unstable. One improvement of the model would be the definition of a test statistic for a more formal delimitation of the limits of the threshold range. It should be noted here that $x_{n}$ is the upper limit for censoring the series, but this does not mean that the values above $x_{n}$ have to be neglected; the exceedances series are constructed from the whole range of the variable, using eq. 1.

The proposed model was applied to the simulated and real data sets to test its performance. The results over the simulated data sets are shown in Table 2. They can be compared with those obtained with the traditional, unique-threshold approach (Table 1). The average quantile estimates were almost identical to the unique-threshold method along the range of threshold values, but the error statistics were greatly reduced. The effect was similar in the two simulation data sets.

The increasing threshold procedure was also applied to the real data sets, using the threshold range limits shown in Table 3. The resulting parameter estimates and quantiles are shown in Table 4 . The quantile estimations were only slightly different than the averages over the range of thresholds using the unique-threshold approach (Table 1), but they have been obtained with a significantly lower number of calculations. A secondary result from Table 3 is the low departure from zero that show the k parameters, except in the cases of Villanúa and 
Panticosa Balneario. This suggest the possibility of using the Exponential distribution instead of GP. In that case the value of $\hat{\kappa}_{x_{0}}^{x_{n}}$ should be set to zero, and proceed with $\hat{\alpha}_{x_{0}}^{x_{n}}$ as explained above.

\section{Conclusions}

Several crucial aspects of the Poisson-GP model for PD series have been addressed in this paper. After declustering, the real data series proved to fit adequately the Poisson-GP model at the different threshold values tested. A tendence to became unstable at the highest thresholds was also detected.

Parameter and quantile estimates have been found to be fairly sensitive to the threshold value chosen. The uncertainty in quantile estimation from series constructed upon a discrete threshold cannot be attributed to the violation of the model assumption. Instead, it is due to natural sampling variability when using low frequency data. The uncertainty of quantile estimates, as demonstrated both by simulated and real data sets, does not depend on the magnitude of the threshold value. Hence a unique optimum threshold value cannot be found.

An alternative procedure has been proposed, based on averaged $\alpha$ and $\kappa$ parameters along an increasing set of threshold values, starting with the lowest value for what the Poisson-GP models holds. Although analytical expressions for variance and errors have not been derived, the estimation errors were reduced by approximately one half in the simulated data set.

Although only the method of PWM has been tested, these results are likely to be independent of the method used for parameter estimation. However, this point needs further research. For example, there are reasons to think that the LH moments introduced by Wang (1997) could also reduce the uncertainty related with the threshold value, as they are less sensitive to the lowest observations of the sample. 


\section{Aknowledgements}

Funding for this research was provided by the following projects: 'Hydrological processes in semi-natural Mediterranean areas' (PROHISEM, REN2001-2268-C02-01/HID), "Hydrological processes in Pirenaic catchments related to the canges in land use and climatic fluctuations" (PIRIHEROS, REN2003-08678/HID), funded by the Spanish CICYT. I want to express my gratitude to three anonymous reviewers, whose comments greatly improved the quality of the initial manuscript. I also wish to acknowledge personal support by a post-doctoral grant funded by the Spanish Government Secretary for Education and Universities and the European Social Fund. 
A)

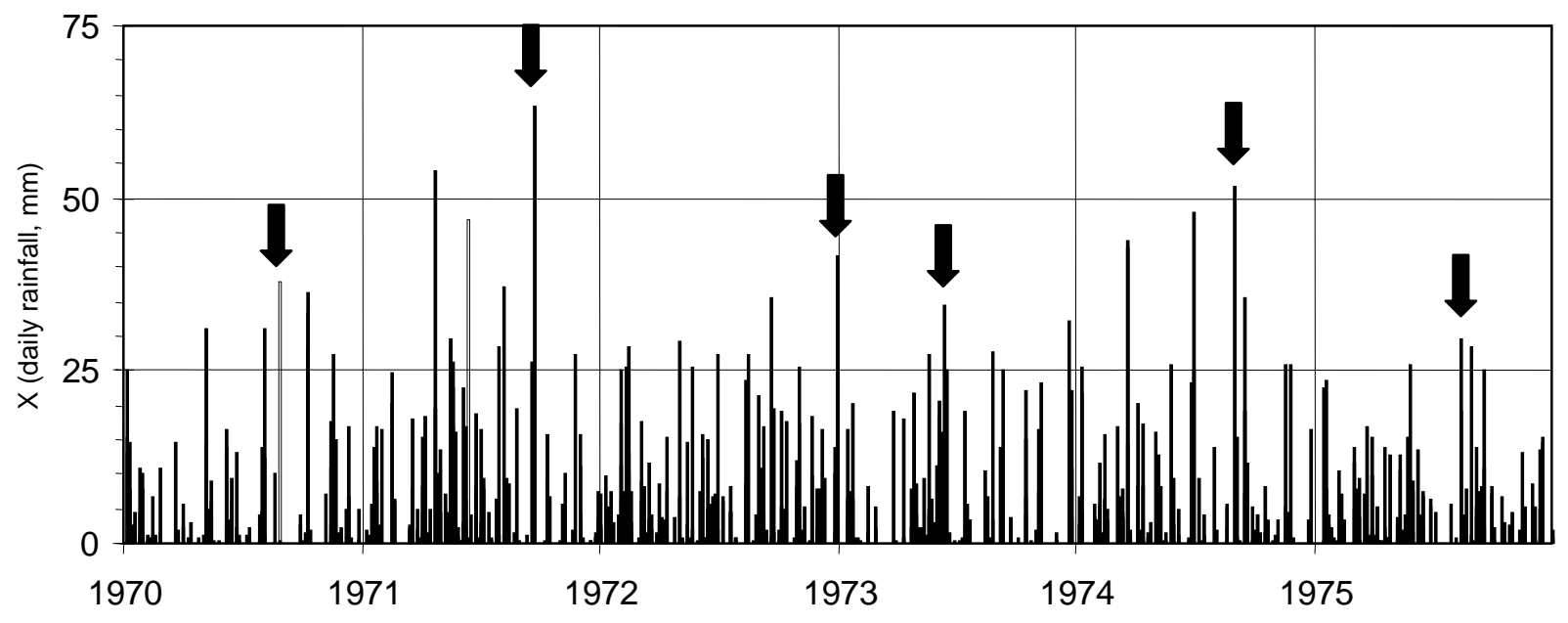

B)

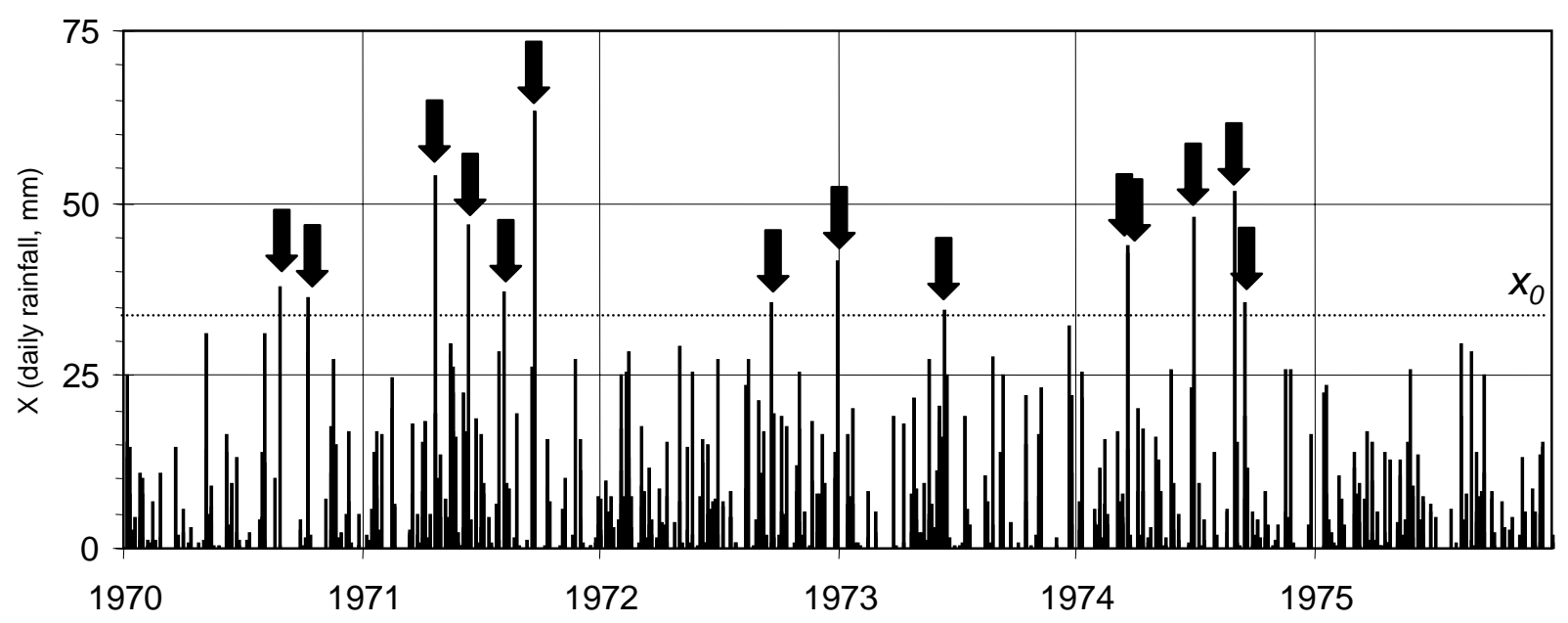




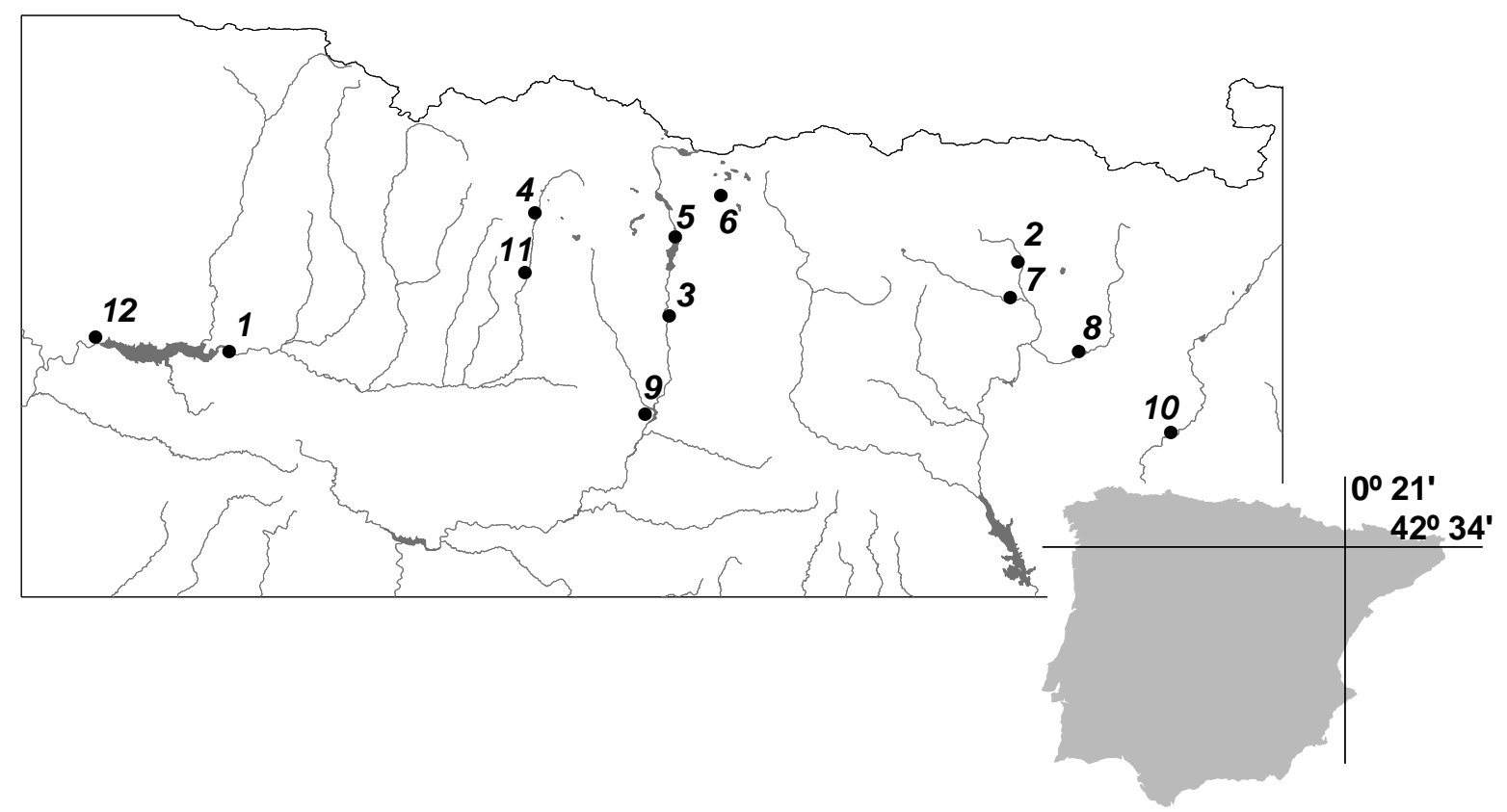


A)

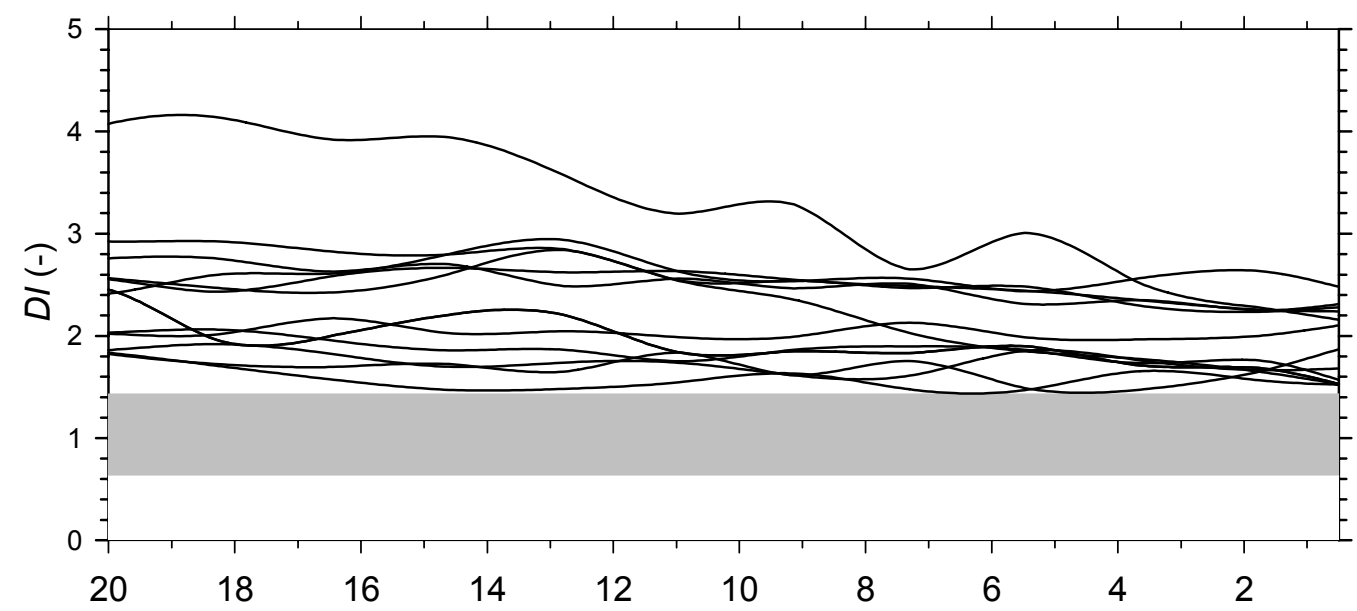

Threshold (occurrences / year)

B)

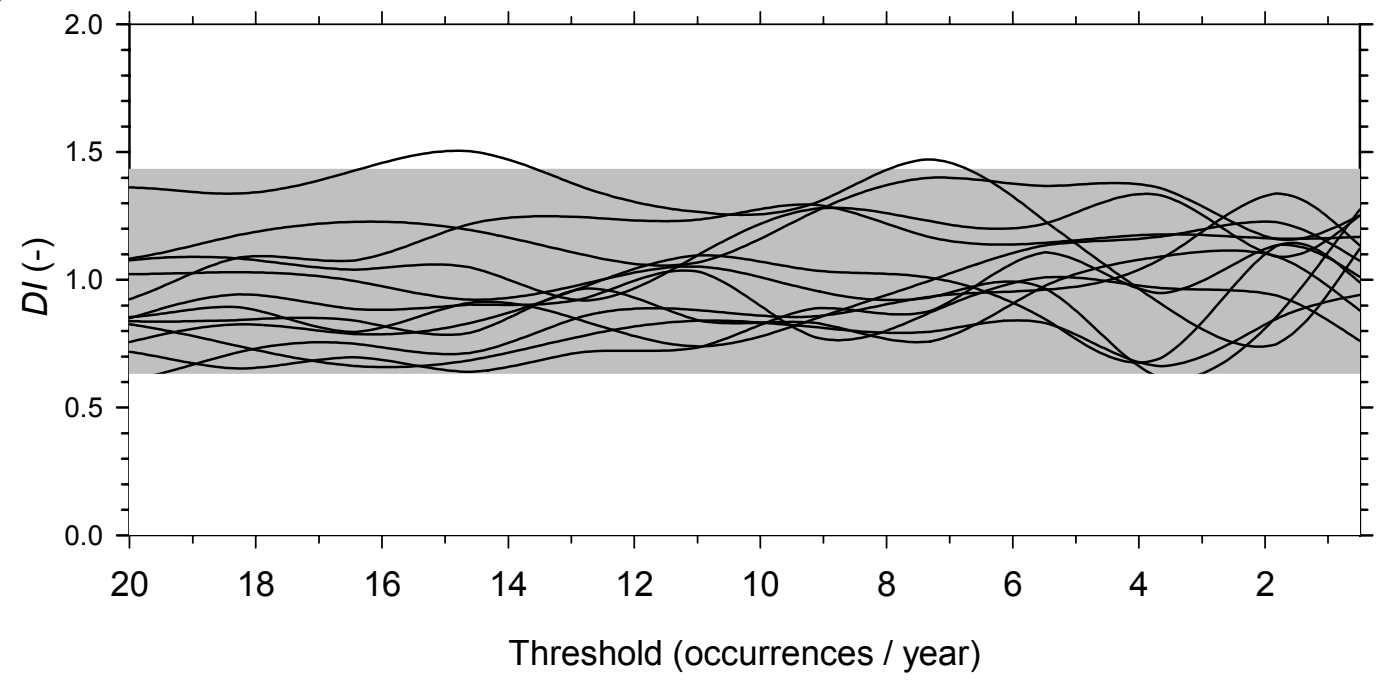


Preprint of article publishzed in Journal of Hydrology, 303:215-230 (2005)
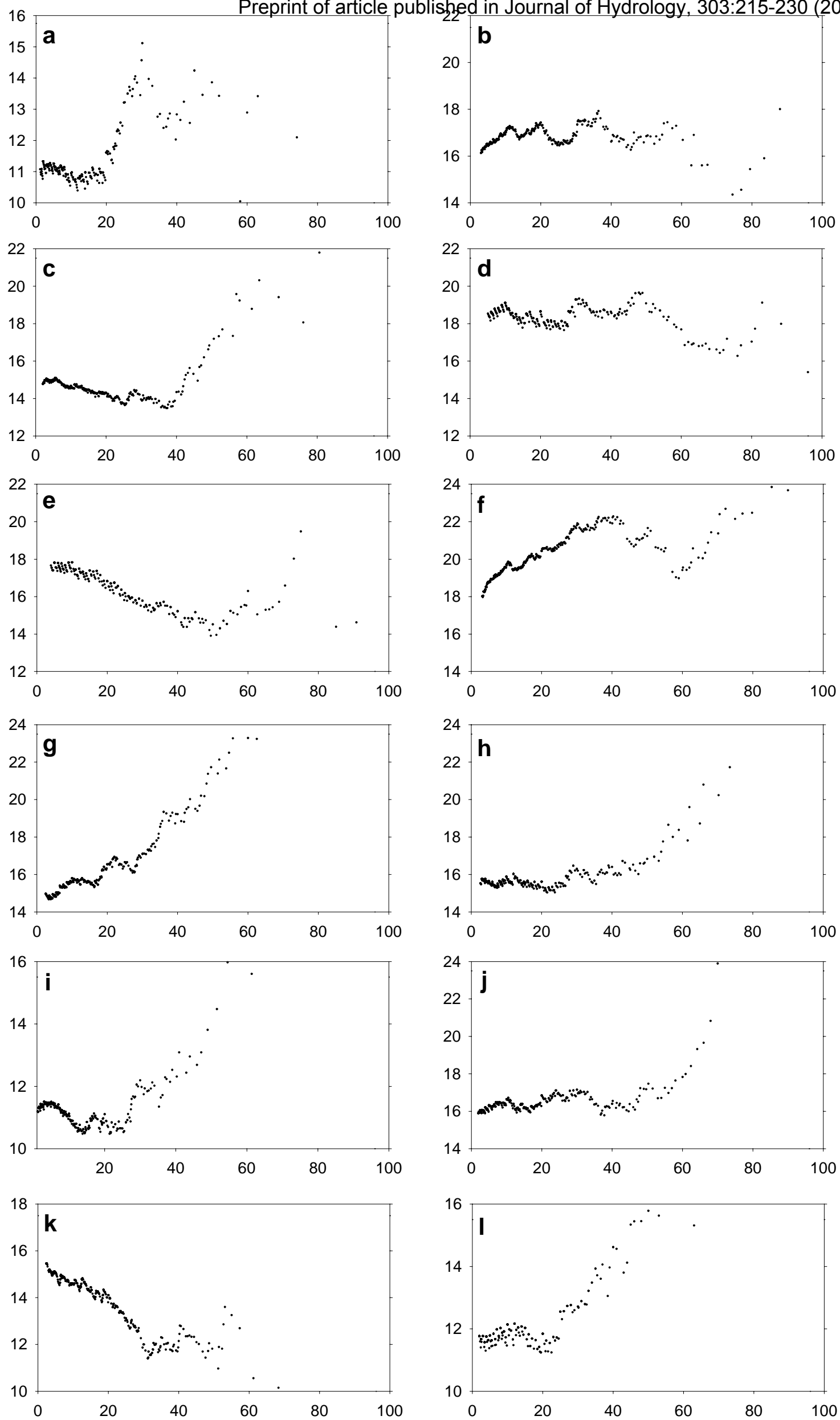
Preprint of article published in Journal of Hydrology, 303:215-230 (2005)

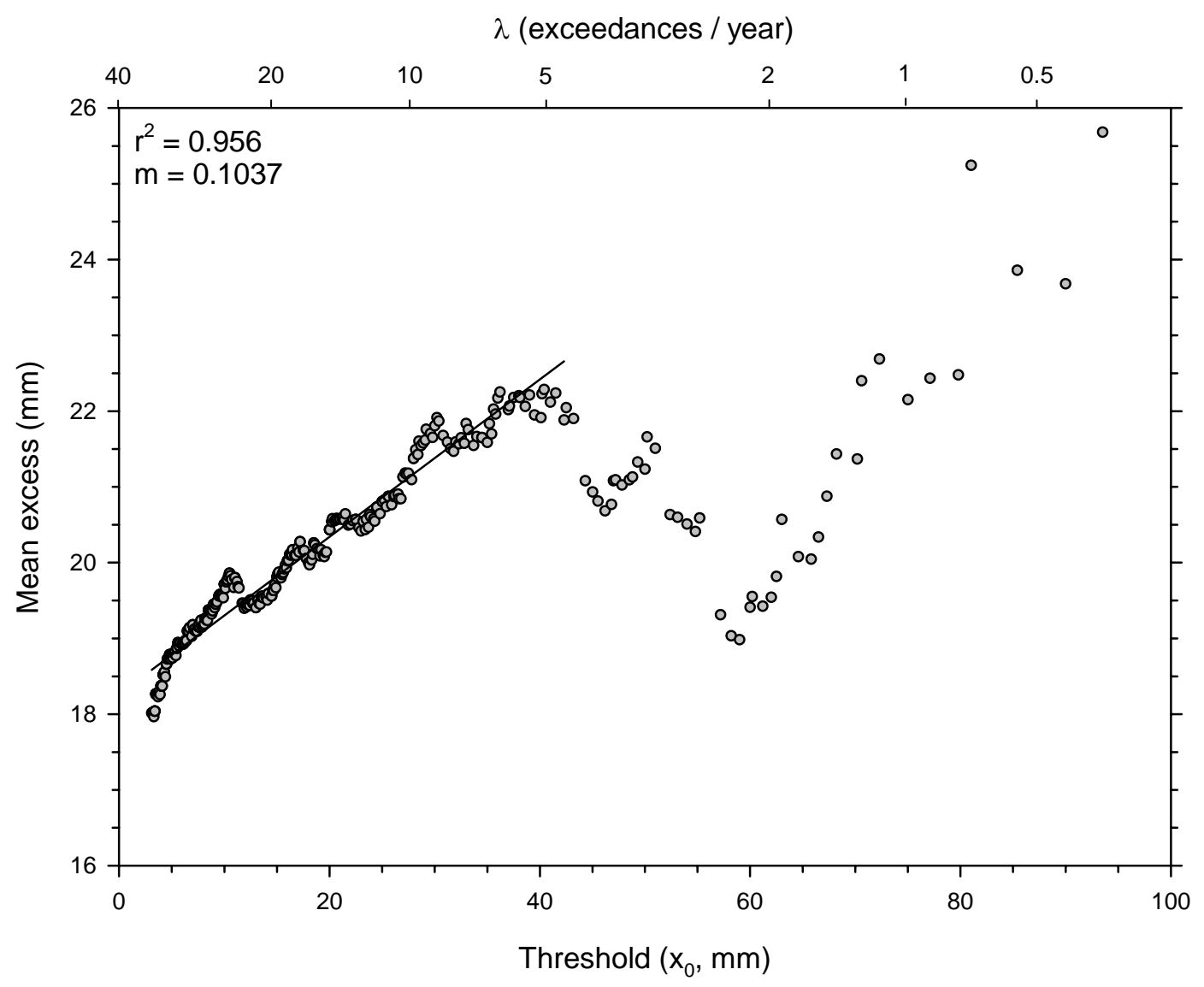



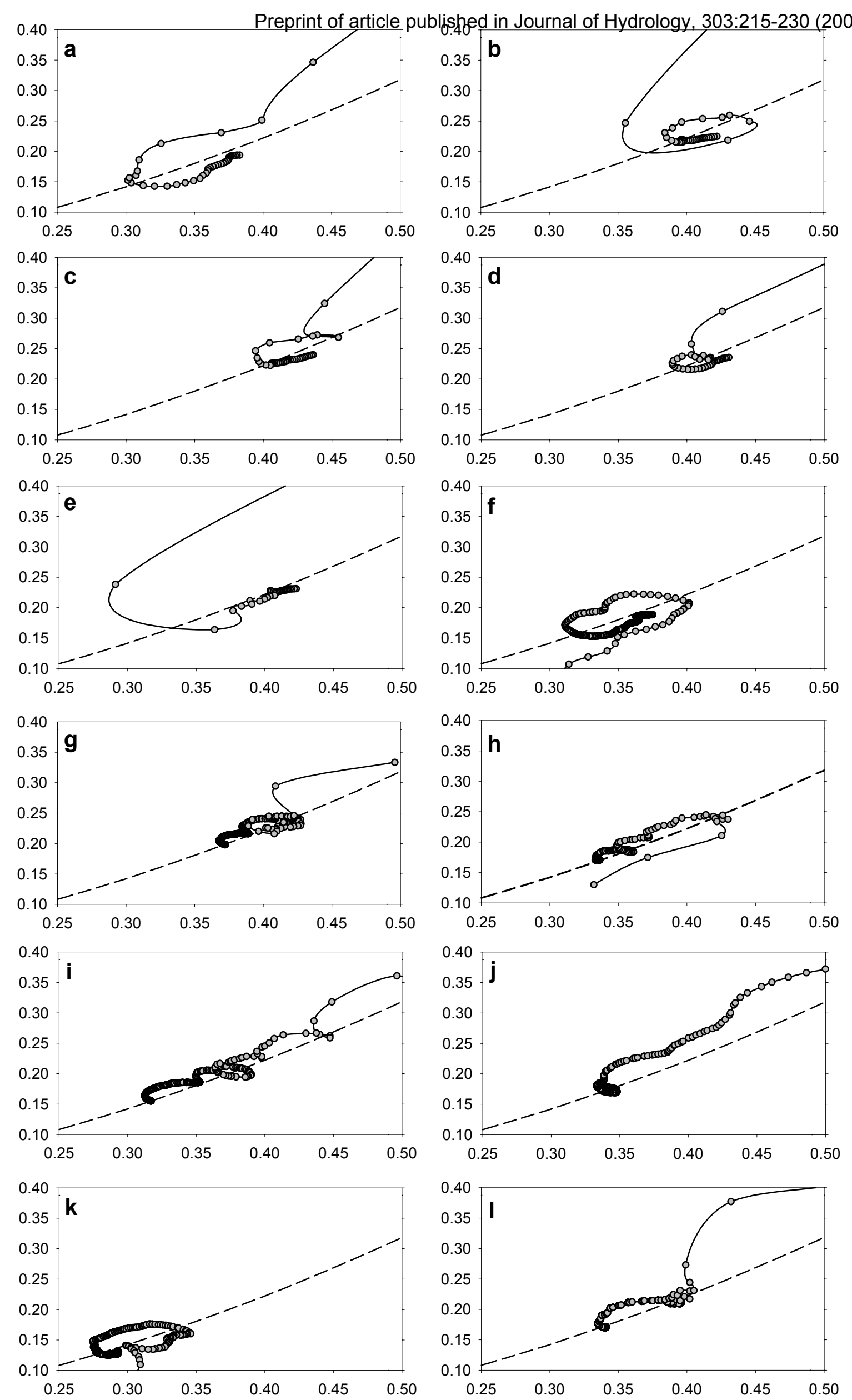
A)

Preprint of article published in Journal of Hydrology, 303:215-230 (2005)

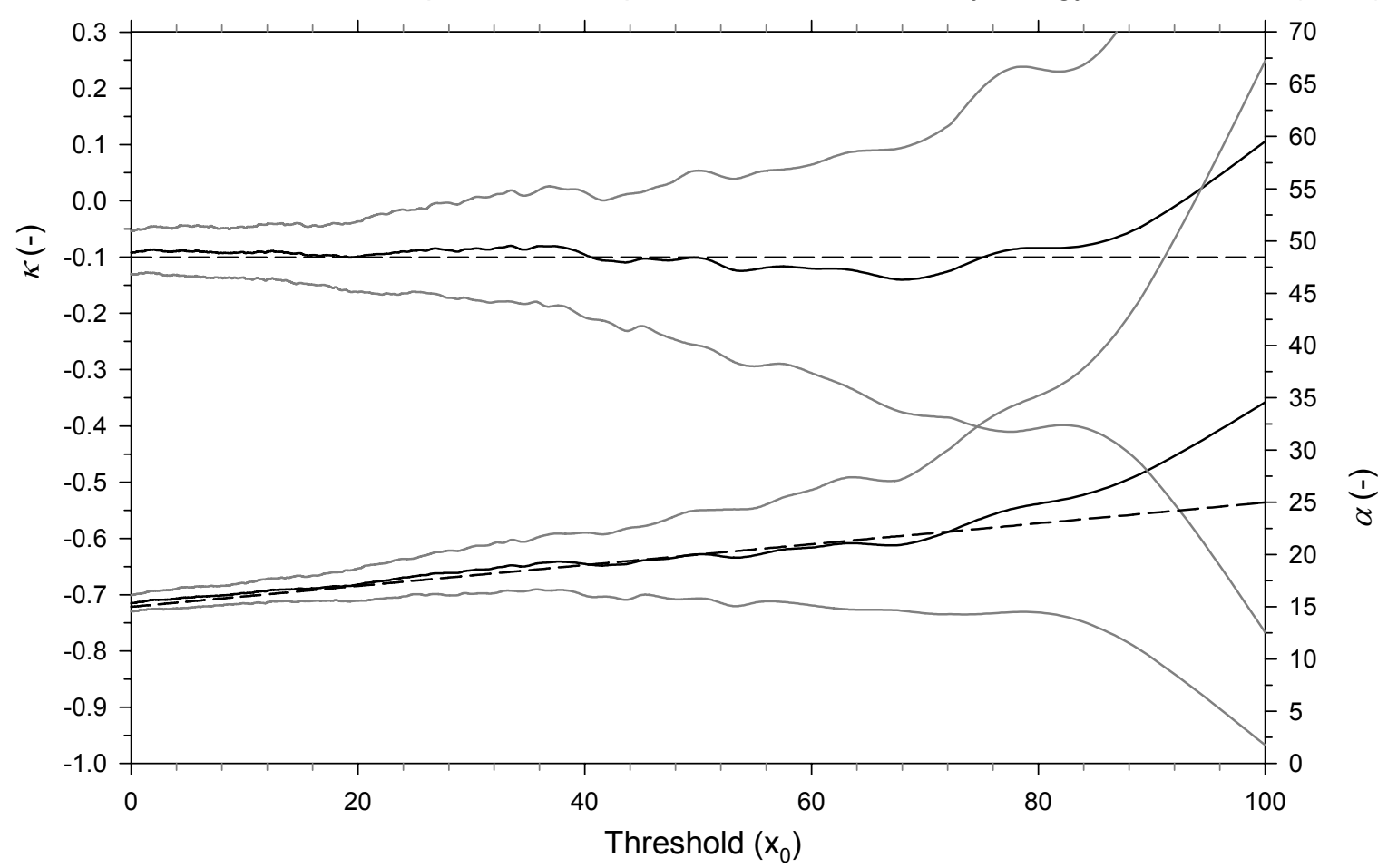

B)

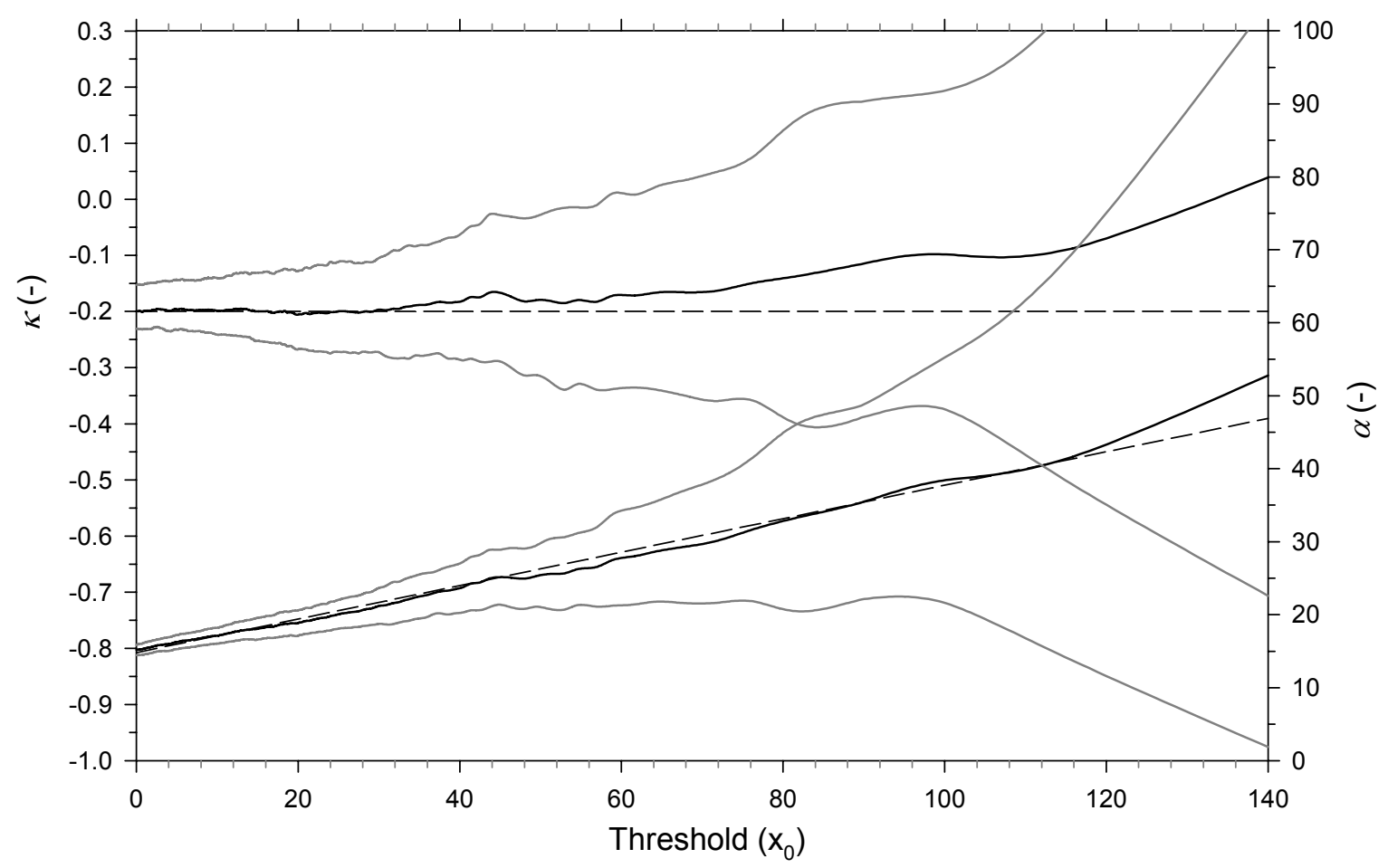


Preprint of article published in Journal of Hydrology, 303:215-230 (2005) $\lambda$ (exceedances / year)

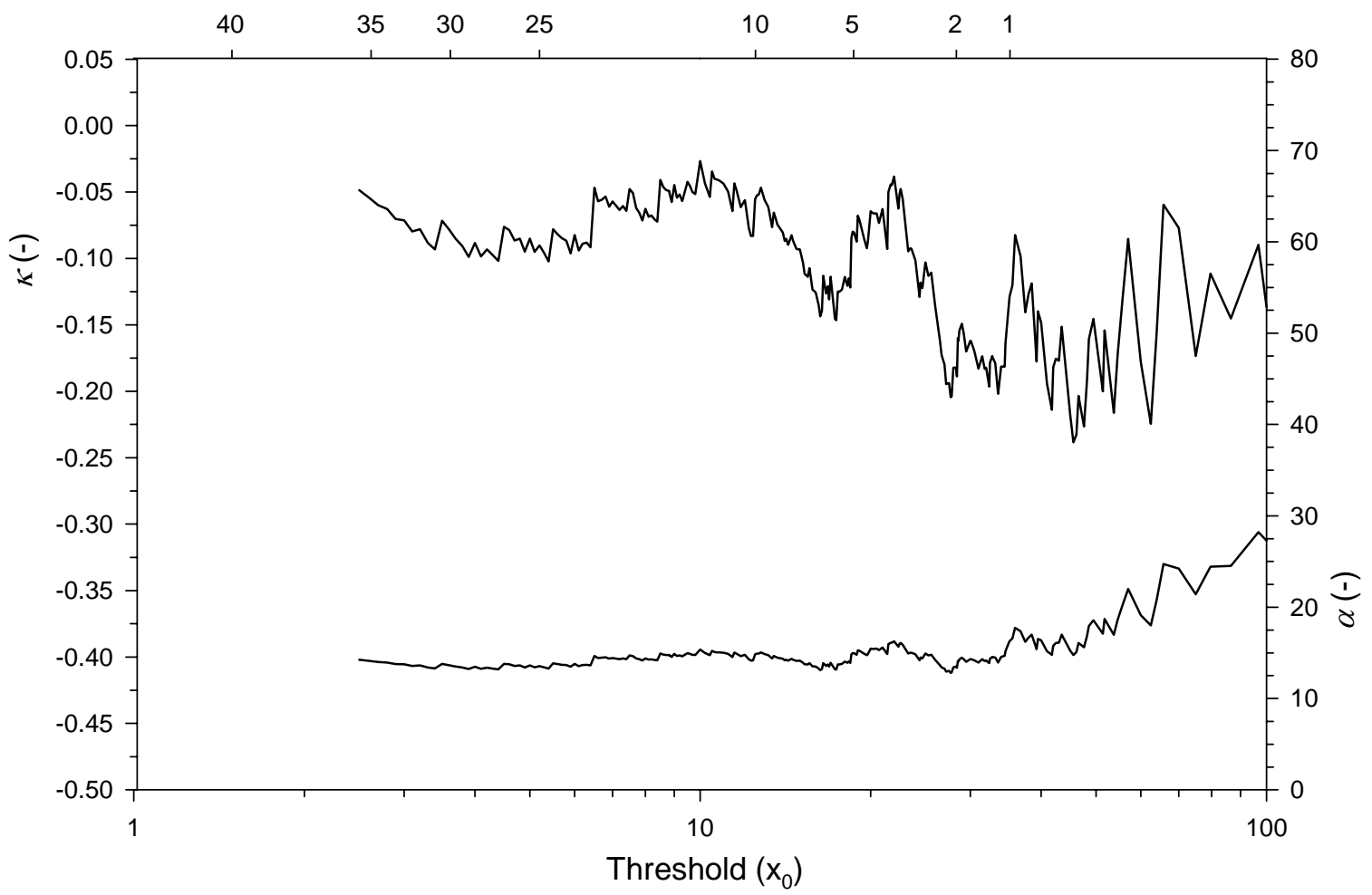


Preprint of article published in Journal of Hydrology, 303:215-230 (2005)

A)

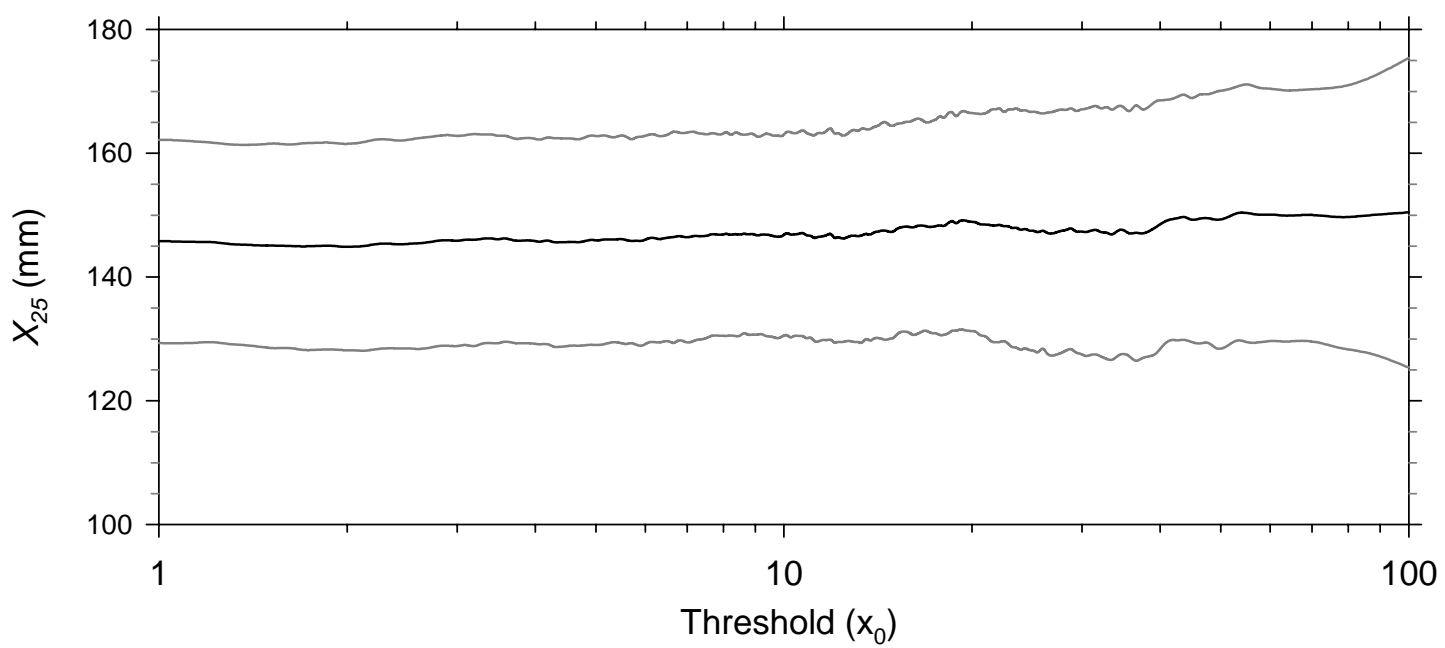

B)

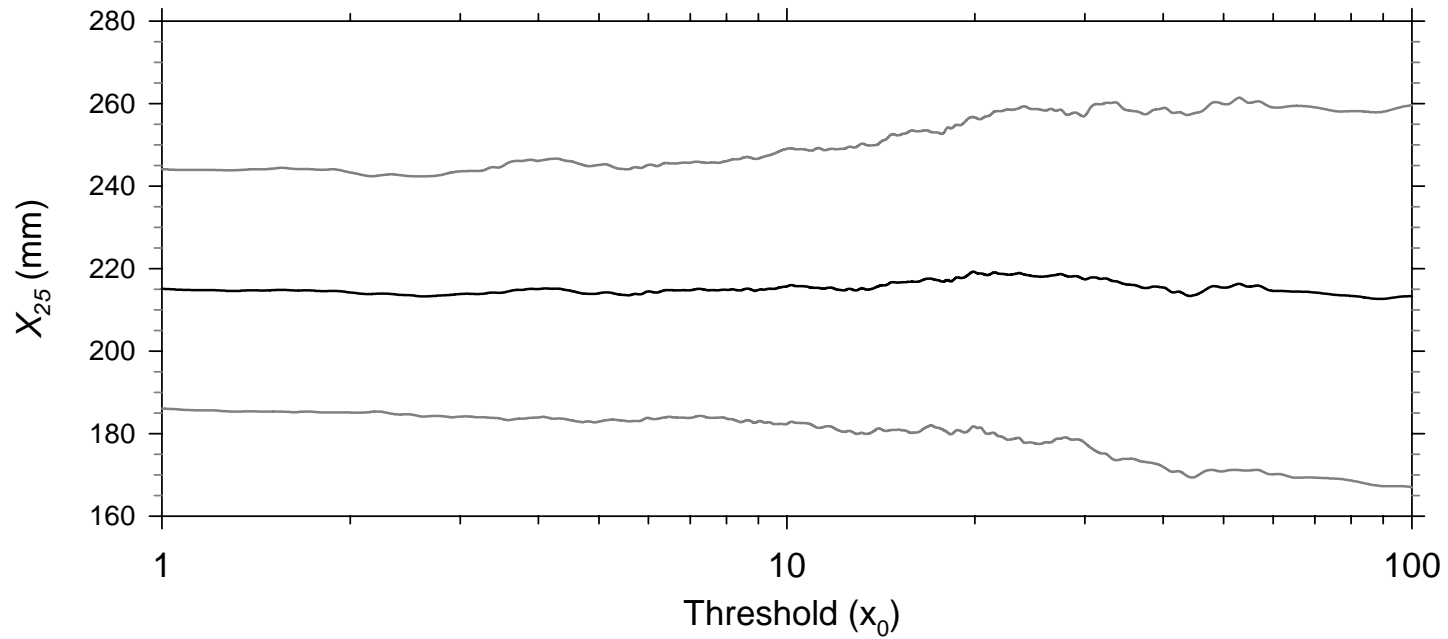

C)

$\lambda$ (exceedances / year)

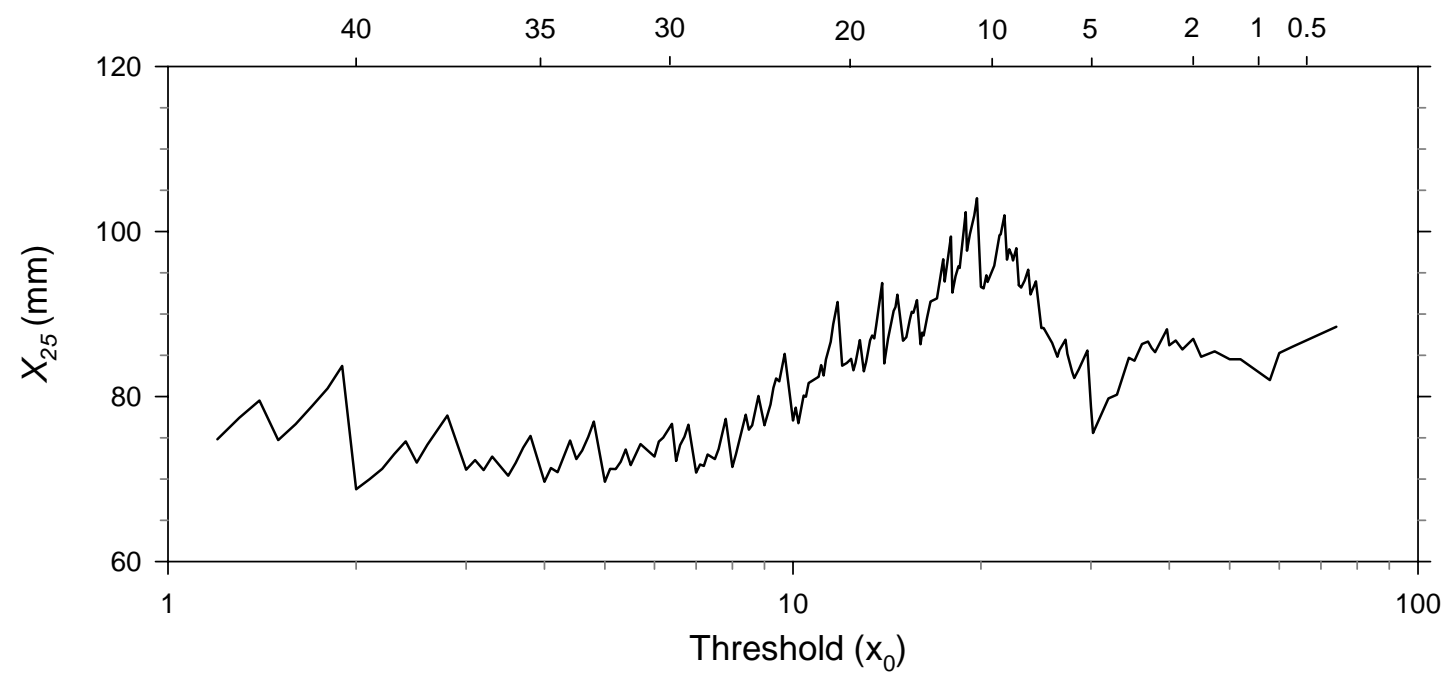


Table 1. Estimation of the 25-year maximum daily rainfall at different truncation levels. Average, maximum and minimum $X_{25}$ estimates and coefficient of variation; standardized mean bias error and root mean square error of the estimates.

\begin{tabular}{|c|c|c|c|c|c|c|c|}
\hline & Station & average $X_{25}$ & $\min X_{25}$ & $\max X_{25}$ & CV & SMBE & RMSE \\
\hline 1 & Artieda & 77.1 & 68.7 & 102.1 & 0.10 & -- & -- \\
\hline 2 & Barrosa & 121.6 & 109.2 & 135.2 & 0.05 & -- & -- \\
\hline 3 & Biescas & 95.4 & 88.7 & 107.7 & 0.04 & -- & -- \\
\hline 4 & Canfranc & 126.1 & 111.6 & 146.2 & 0.06 & -- & -- \\
\hline 5 & Panticosa Balneario & 101.3 & 93.3 & 112.8 & 0.04 & -- & -- \\
\hline 6 & Pineta & 154.7 & 131.5 & 165.6 & 0.05 & -- & -- \\
\hline 7 & Plandescún & 127.9 & 116.1 & 146.8 & 0.06 & -- & -- \\
\hline 8 & Pueyo de Jaca & 107.6 & 98.3 & 121.9 & 0.05 & -- & -- \\
\hline 9 & Sabiñánigo & 72.5 & 65.7 & 87.2 & 0.07 & -- & -- \\
\hline 10 & Seira & 116.0 & 106.1 & 125.9 & 0.04 & -- & -- \\
\hline 11 & Villanúa & 84.6 & 77.0 & 96.8 & 0.04 & -- & -- \\
\hline 12 & Yesa & 78.6 & 72.9 & 95.1 & 0.06 & -- & -- \\
\hline \multicolumn{8}{|c|}{ Poisson/GP, $\kappa=0$, PWM } \\
\hline \multicolumn{2}{|c|}{ Poisson/GP, $\kappa=-0.1$, PWM } & 147.0 & -- & -- & -- & 0.003 & 16.202 \\
\hline \multicolumn{2}{|c|}{ Poisson/GP, $\kappa=-0.2$, PWM } & 215.6 & -- & -- & -- & -0.012 & 29.668 \\
\hline
\end{tabular}


Preprint of article published in Journal of Hydrology, 303:215-230 (2005)

Table 2. Estimation of the 25-year maximum daily rainfall with the increasing threshold approach, simulated data. Average, maximum and minimum $X_{25}$ estimates and coefficient of variation; standardized mean bias error and root mean square errors of the estimates.

\begin{tabular}{lccc}
\hline & average $\boldsymbol{X}_{\mathbf{2 5}}$ & SMBE & RMSE \\
\hline Poisson/GP, $\boldsymbol{\kappa}=\mathbf{- 0 . 1}$, PWM & 146.9 & 0.002 & 12.528 \\
Poisson/GP, $\boldsymbol{\kappa}=\mathbf{- 0 . 2}$, PWM & 215.7 & -0.011 & 24.210 \\
\hline
\end{tabular}


Table 3. Lowest and highest limits $\left(x_{0}, \mathrm{~mm} ; \lambda\right.$, exceedances / year $)$

for the range of thresholds used with the real data series.

Preprint of article published in Journal of Hydrology, 303:215-230 (2005)

\begin{tabular}{cccccc}
\hline & Station & $\boldsymbol{x}_{0}$ & $\boldsymbol{\lambda}_{\boldsymbol{0}}$ & $\boldsymbol{x}_{\boldsymbol{n}}$ & $\boldsymbol{\lambda}_{n}$ \\
\hline $\mathbf{1}$ & Artieda & 1.2 & 41.17 & 19.7 & 7.70 \\
$\mathbf{2}$ & Barrosa & 3.0 & 39.99 & 36.0 & 5.11 \\
$\mathbf{3}$ & Biescas & 2.0 & 39.37 & 38.9 & 3.17 \\
$\mathbf{4}$ & Canfranc & 5.1 & 39.79 & 46.5 & 4.04 \\
$\mathbf{5}$ & Panticosa Balneario & 4.0 & 39.68 & 51.0 & 2.54 \\
$\mathbf{6}$ & Pineta & 3.1 & 39.50 & 42.5 & 4.70 \\
$\mathbf{7}$ & Plandescún & 2.5 & 39.54 & 39.3 & 3.30 \\
$\mathbf{8}$ & Pueyo de Jaca & 2.6 & 39.84 & 42.0 & 3.06 \\
$\mathbf{9}$ & Sabiñánigo & 1.2 & 39.13 & 26.0 & 4.11 \\
$\mathbf{1 0}$ & Seira & 2.0 & 39.51 & 46.0 & 2.62 \\
$\mathbf{1 1}$ & Villanúa & 2.3 & 41.02 & 32.1 & 6.26 \\
$\mathbf{1 2}$ & Yesa & 2.0 & 38.13 & 24.0 & 5.64 \\
\hline
\end{tabular}


Table 4. Parameter estimates and maximum daily rainfall for a 25 years return period, using the increasing threshold approach.

\begin{tabular}{ccccc}
\hline & Station & $\hat{\alpha}_{x_{0}}^{X_{n}}$ & $\hat{\boldsymbol{\kappa}}_{x_{0}}^{X_{n}}$ & $X_{25 x_{0}}^{x_{n}}$ \\
\hline $\mathbf{1}$ & Artieda & 10.995 & -0.003 & 77.4 \\
$\mathbf{2}$ & Barrosa & 16.596 & -0.009 & 120.4 \\
$\mathbf{3}$ & Biescas & 15.218 & 0.026 & 97.8 \\
$\mathbf{4}$ & Canfranc & 19.469 & 0.029 & 126.1 \\
$\mathbf{5}$ & Panticosa Balneario & 20.121 & 0.106 & 102.1 \\
$\mathbf{6}$ & Pineta & 18.022 & -0.058 & 154.8 \\
$\mathbf{7}$ & Plandescún & 13.420 & -0.086 & 127.8 \\
$\mathbf{8}$ & Pueyo de Jaca & 15.763 & 0.005 & 108.9 \\
$\mathbf{9}$ & Sabiñánigo & 11.721 & 0.031 & 73.6 \\
$\mathbf{1 0}$ & Seira & 16.069 & -0.010 & 116.0 \\
$\mathbf{1 1}$ & Villanúa & 17.136 & 0.110 & 84.9 \\
$\mathbf{1 2}$ & Yesa & 12.260 & 0.025 & 79.3 \\
\hline
\end{tabular}

